\title{
Resuscitative therapy with erythropoietin reduces oxidative stress and inflammatory responses of vital organs in a rat severe fixed-volume hemorrhagic shock model
}

\author{
Mina Ranjbaran ${ }^{1}$, Mehri Kadkhodaee ${ }^{1}$, Behjat Seifi ${ }^{1}$, Reza Mirzaei ${ }^{2}$ and Parisa Ahghari ${ }^{3}$ \\ ${ }^{1}$ Department of Physiology, Faculty of Medicine, Tehran University of Medical Sciences, Tehran, Iran \\ ${ }^{2}$ Department of Immunology, Faculty of Medicine, Tehran University of Medical Sciences, Tehran, Iran \\ ${ }^{3}$ Department of Physiology, Faculty of Medicine, Hamedan University of Medical Sciences, Hamedan, Iran
}

\begin{abstract}
Hemorrhagic shock (HS) still has a high mortality rate and none of the known resuscitative regimens completely reverse its adverse outcomes. This study investigated the effects of different models of resuscitative therapy on the healing of organ damage in a HS model. Male Wistar rats were randomized into six groups: Sham, without HS induction; HS, without resuscitation; $\mathrm{HS}+\mathrm{Blood}$, resuscitation with the shed blood; HS+Blood+NS, resuscitation with blood and normal saline; HS+Blood+RL, resuscitation with blood and Ringer's lactate; EPO, erythropoietin was added to the blood and RL. Blood and urine samples were obtained $3 \mathrm{~h}$ after resuscitation. Kidney, liver and brain tissue samples were harvested for multiple organ failure evaluation. Survival rate was the highest in the Sham, EPO and HS+Blood+RL groups compared to others. Plasma creatinine concentration, ALT, AST, urinary NAG activity and renal NGAL mRNA expression significantly increased in the HS+Blood+RL group compared to the Sham group. There was a significant increase in tissue oxidative stress markers and pro-inflammatory cytokines in HS+Blood+RL group compared to the Sham rats. EPO had more protective effects on multiple organ failure compared to the HS+Blood+RL group. EPO, as a resuscitative treatment, attenuated HS-induced organ damage. It seems that it has a potential to be attractive for clinical trials.
\end{abstract}

Key words: Hemorrhagic shock - Resuscitation - Erythropoietin - Multiple organ failure Pro-inflammatory cytokines

\begin{abstract}
Abbreviations: Cr, creatinine; EPO, erythropoietin; HS, hemorrhagic shock; IL-6, interleukin-6; MDA, malondialdehyde; NAG, N-acetyl- $\beta$-D-glucosaminidase; NGAL, neutrophil gelatinaseassociated lipocalin; NS, normal saline; RL, Ringer's lactate; SOD, superoxide dismutase; TNF- $\alpha$, tumor necrosis factor- $\alpha$.
\end{abstract}

\section{Introduction}

Shock is a functional defect in the circulatory system and hemorrhagic shock (HS) is one of the most frequent types (Fülöp et al. 2013). In the HS state, there is a serious amount of blood loss which leads to insufficient tissue perfusion and reduced oxygen supply. This causes microcirculatory failure, breakdown of cell function and, eventually, multiple organ

Correspondence to: Mehri Kadkhodaee, Department of Physiology, Faculty of Medicine, Tehran University of Medical Sciences, Tehran, Iran

E-mail: kadkhodm@tums.ac.ir failure syndrome (Boldt 2004; Knotzer et al. 2006). Mortality may be directly as a result of considerable blood loss or take place indirectly due to multiple organ failure (Ronn et al. 2011).

Although volume resuscitation is the main therapeutic goal in HS, aggressive restoration of intravascular volume leads to increased mortality. Different regimens have been suggested (Knotzer et al. 2006; Harlak et al. 2009; Ronn et al. 2011) but none completely reverse the microcirculatory dysfunction following serious blood loss (Harlak et al. 2009). Thus, suitable fluid resuscitation and contribution of these fluids to the healing of damaged organs are mandatory to eliminate the detrimental effects of severe and prolonged 
hypo-perfusion. Accordingly, we examined the effects of different resuscitative fluids on the healing of organ damage in a rat HS model.

This preclinical trial is based on small animals study. In this study, considering the advantages and disadvantages of the accessible HS models, severe and life-threatening HS was induced by a fixed-volume model in rats. This model is very similar to HS occurrence in human clinical practice and represents the majority of emergency conditions realistically. Fixed-volume models are based on body weight (Ronn et al. 2011). In these models, a predetermined percentage of the total calculated blood volume is withdrawn over time according to the observer settings (Fülöp et al. 2013).

Erythropoietin (EPO), a well-known hormone for red blood cell proliferation, has been reported to protect organs and tissues against ischemia-reperfusion injuries. Recently, it is administered in low-flow states such as HS and has shown beneficial effects in this condition (Borovnik-Lesjak et al. 2014). Therefore, erythropoietin was added into the resuscitative fluid to evaluate its protective effects on organs following HS.

The objectives of this study were to 1) set up a fixedvolume model of HS, 2) compare the effects of different resuscitative regimens on $\mathrm{HS}$ outcome and survival rate and 3) investigate multiple organ failure in HS and resuscitation by evaluating the pro-inflammatory cytokines and oxidative stress indices in different organs.

\section{Materials and Methods}

\section{Animals}

Male Wistar rats weighing 285-300 g were used. All procedures and animal care methods in the experiments were approved by the Animal Ethics Committee of Tehran University of Medical Sciences. The rats were maintained under standard conditions ( $12 \mathrm{~h}$ light-dark cycle; $20-22^{\circ} \mathrm{C}$ ) and had free access to food and water.

\section{Anesthesia and catheter placement}

Rats were anesthetized using ketamine $(50 \mathrm{mg} / \mathrm{kg}$ ) and xylazine $(10 \mathrm{mg} / \mathrm{kg})$ administered intraperitoneally. Additional ketamine $(20 \mathrm{mg} / \mathrm{kg}$, i.p.) was injected if needed. After performing a sterile incision in the left groin, two catheters were placed within the femoral artery and vein. Thereafter, another catheter was inserted into the trachea to facilitate respiration.

\section{Induction of hemorrhagic shock and resuscitation}

After catheter insertion, HS was induced by removing fifty percent of the total calculated blood volume via femoral artery into heparinized syringes. Blood removal took approximately 30 minutes. Fifty percent of total blood volume was calculated by the following formula: animal weight [g] $\times 0.03+0.7 \mathrm{ml}$ (Ahmadi-Yazdi et al. 2009) .

Two hours after shock induction, group-specific resuscitative fluids were infused for $30 \mathrm{~min}$ into the left femoral vein by a heparinized syringe. Animals were closely monitored throughout the experimental period up to $3 \mathrm{~h}$ after resuscitation.

\section{Experimental design}

Animals were randomized into six experimental groups: 1) Sham, anesthesia and surgery without HS induction; 2) HS, hemorrhagic shock without resuscitation; 3) HS+blood, resuscitated with the total volume of removed blood; 4) HS+blood+NS, resuscitation was performed with collected blood plus normal saline (NS) at twice the volume of the shed blood; 5) HS+blood+RL, resuscitated with collected blood plus Ringer's lactate (RL) at twice the volume of the shed blood; and 6) EPO group, erythropoietin (EPO) (300 IU/kg) was added to the shed blood and RL for resuscitation. In this group, the withdrawn blood was mixed gently with the NS or RL immediately before resuscitation, and was filtered through a neonatal blood filter to avoid infusion of microclots, which may have formed during blood storage.

\section{Assessment of plasma parameters}

At the end of each procedure, blood samples were taken via femoral artery into heparinized syringes and plasma was obtained by centrifugation $(3500 \times g$ for $10 \mathrm{~min})$ and stored at $-70^{\circ} \mathrm{C}$ until further use. Plasma concentration of creatinine ( $\mathrm{Cr}$ ) was determined as an indicator of renal functional decline by using a fully automated clinical chemistry analyzer (Hitachi 704 auto-analyzer, Japan). Liver injury was assessed by plasma levels of alanine aminotransferase (ALT, a specific marker for hepatic parenchymal injury) and aspartate aminotransferase (AST, a non-specific marker for hepatic injury).

\section{Assessment of urinary NAG and Cr concentration}

Urine was collected at the end stage of each experiment and $\mathrm{N}$-acetyl- $\beta$-D-glucosaminidase (NAG) activity and $\mathrm{Cr}$ concentration were measured. The spectrophotometric assay for urinary NAG activity is based on the enzymatic hydrolysis of p-nitrophenyl-N- $\beta$ - D-acetyl-glucosaminide as substrate (Seifi et al. 2014). Urinary Cr level was evaluated with the use of fully automated clinical chemistry analyzer (Hitachi 704 auto-analyzer, Japan). To show less variability, urinary NAG values should be presented as a ratio to urinary $\mathrm{Cr}$ concentration (Ali et al. 2014). 
Assessment of renal NGAL mRNA expression by Real-time PCR

Total RNA was isolated from renal tissue samples according to the manufacturer's instructions (RNeasy Mini Kit; Qiagen). RNA content and purity were measured using the Nanodrop 2000 (Thermo- Scientific, USA). The cDNA was synthesized from $4 \mu \mathrm{g}$ of total RNA using the PrimeScript RT Master Mix (Takara, Japan) as instructed and was stored at $-70^{\circ} \mathrm{C}$. Real-time PCR amplifications were carried out using the ABI 7500 system (Applied Biosystems, USA). The reaction mixture contained $4 \mu \mathrm{l}$ of diluted cDNA, $5 \mathrm{pm}$ of each primer and $10 \mu \mathrm{l}$ of $2 X$ SYBR green master mixes in a total volume of $20 \mu \mathrm{l}$.

The amount of mRNA for neutrophil gelatinase-associated lipocalin $(N G A L)$ gene was normalized by using HPRT-1 (hypoxanthine phosphoribosyltransferase 1). PCR primers for the two analyzed genes are shown as follows:

NGAL gene (Sense Strand Sequence): CTCTGTTCCCACCGACCAAT

NGAL gene (Anti-Sense Strand Sequence): TTGGTGGAATCATGGCTGGT

HPRT-1 gene (Sense Strand Sequence): CTCCTCAGACCGCTTTTCCC

HPRT-1 gene (Anti-Sense Strand Sequence): AGCAAGTCTTTCAGTCCTGTCC

\section{Histopathologic analysis of organs}

Kidney and liver tissue samples were fixed in 10\% formaldehyde and then paraffin-embedded sections $(4 \mu \mathrm{m}$ thicknesses) were stained with hematoxylin-eosin. The histopathologic changes were documented photographically.

\section{Tissue oxidative stress indices}

Kidney, liver and brain tissue samples were harvested and stored at $-70^{\circ} \mathrm{C}$ after being snap-frozen in liquid nitrogen. Malondialdehyde (MDA) content and superoxide dismutase (SOD) activity were evaluated in the supernatant of tissue homogenates.

A spectrophotometric method was used to evaluate MDA level and then the optical density of supernatants was measured at $532 \mathrm{~nm}$ (Esterbauer and Cheeseman 1990).

SOD activity of the tissue samples were determined based on the method by Paoletti and Mocali (1988). SOD activity evaluation is based on the inhibition of superoxide-induced NADPH oxidation. The optical density of supernatants was measured at $340 \mathrm{~nm}$.

\section{Tissue pro-inflammatory cytokines assay by ELISA}

Tumor necrosis factor- $\alpha$ (TNF- $\alpha$ ) and interleukin-6 (IL-6) concentrations were determined in the kidney, liver and brain tissue samples separately by enzyme-linked immunosorbent assay (ELISA). All reagents, working standards and samples were brought to room temperature and prepared according to the manufacturer's directions (R\&D Systems, Inc., Minneapolis, MN). The tissue samples were homogenized in PBS briefly and centrifuged at $3000 \mathrm{rpm}$ for $15 \mathrm{~min}$ at $4^{\circ} \mathrm{C}$. The supernatant was collected for assessment of TNF- $\alpha$ and IL-6. Using an ELISA reader (BioTek Instrument (ELX $800)$, Inc, USA), optical density of reactions was measured at a wavelength of $450 / 570 \mathrm{~nm}$.

\section{Statistical analysis}

The data were presented as mean \pm standard error of mean. Chi-square and Fisher's exact tests were used for survival rate analysis. Comparisons between groups were made using one way ANOVA and Tukey's post-hoc test; $p<0.05$ was considered statistically significant.

\section{Results}

Effects of different resuscitative regimens on survival rate in HS state

No mortality was observed in the Sham group. In the absence of resuscitation (HS group), $90 \%$ of the animals expired within predetermined experimental time (of ten rats, only one rat survived, Fig. 1). In the HS+Blood group, 92.3\% of the animals expired before the end of protocol (of thirteen rats, only one rat survived). In the HS+Blood+NS group, the survival rate was $16.66 \%$ (of twelve rats, only two rats survived). The survival

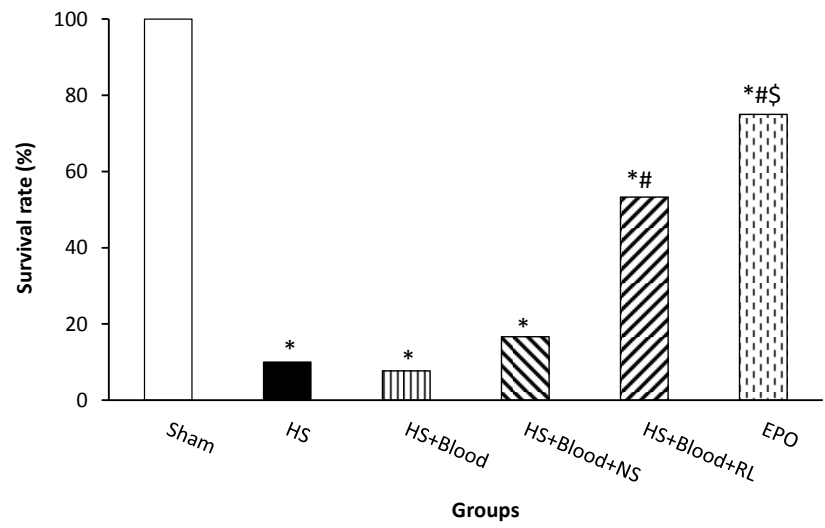

Figure 1. Effects of different resuscitative regimens on survival rate in hemorrhagic shock. Data are presented as percent. HS, hemorrhagic shock; NS, normal saline; RL, Ringer's lactate; EPO, erythropoietin. ${ }^{\star} p<0.05 v s$. Sham group; ${ }^{\#} p<0.05 v s$. HS, HS+Blood, HS+Blood+NS groups; ${ }^{\$} p<0.05$ vs. HS+Blood+RL group. For more information, see Materials and Methods. 
Table 1. Effects of severe hemorrhagic shock and resuscitation with blood + Ringer's lactate with or without erythropoietin on plasma markers in rats

\begin{tabular}{lccc}
\hline & \multicolumn{3}{c}{ Group } \\
\cline { 2 - 4 } & Sham & HS+Blood+RL & EPO \\
\hline Plasma Cr $(\mathrm{mg} / 100 \mathrm{ml})$ & $0.61 \pm 0.03$ & $1.6 \pm 0.12^{\star}$ & $0.81 \pm 0.02^{\star \#}$ \\
\hline ALT $(\mathrm{U} / \mathrm{l})$ & $71.33 \pm 20.45$ & $1046 \pm 146.17^{\star}$ & $435 \pm 103.59^{* \#}$ \\
AST $(\mathrm{U} / \mathrm{l})$ & $93.33 \pm 25.71$ & $1433.66 \pm 160.02^{\star}$ & $495 \pm 97.01^{\star \#}$ \\
\hline
\end{tabular}

Data are presented as the mean \pm standard error of mean (SEM). HS, hemorrhagic shock; RL, Ringer's lactate; EPO, erythropoietin; Cr, creatinine; ALT, alanine aminotransferase; AST, aspartate aminotransferase. ${ }^{*} p<$ 0.05 vs. Sham group, $\# p<0.05$ vs. HS+Blood+RL group. For more information, see Materials and Methods.

rate analysis showed that rats in the HS+Blood+RL group had a significantly greater survival rate (53.3\%) compared to those in the other groups (of fifteen rats, eight rats survived). The highest survival rate (75\%) was observed in the EPO group (of eight rats, six rats survived). Most of the rats expired within $1.5 \mathrm{~h}$ of resuscitation in $\mathrm{HS}, \mathrm{HS}+\mathrm{Blood}$ and $\mathrm{HS}+\mathrm{Blood}+\mathrm{NS}$ groups. Thus, all subsequent data analysis was performed between Sham, HS+Blood+RL and EPO groups.

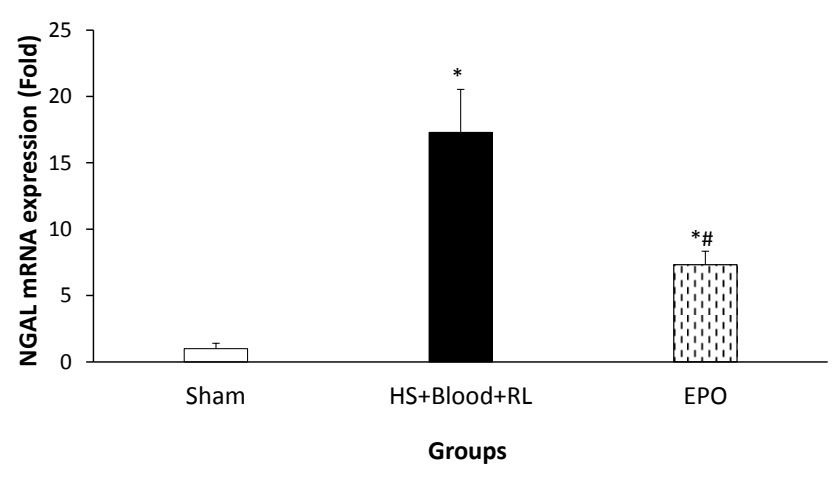

Figure 2. Effects of severe hemorrhagic shock and resuscitation with blood + Ringer's lactate with or without erythropoietin on renal NGAL mRNA expression. Data are presented as the mean \pm SEM. NGAL, neutrophil gelatinase-associated lipocalin. ${ }^{*} p<0.05 v s$. Sham group; $\# p<0.05 v s$. HS+Blood+RL group. For more information, see Materials and Methods.
Plasma Cr concentration as an indicator of kidney function in HS state

$\mathrm{HS}+\mathrm{Blood}+\mathrm{RL}$ rats showed significant increase in plasma $\mathrm{Cr}$ concentration compared to the Sham group (Table 1). In the EPO group, plasma Cr concentration significantly decreased compared to the HS+Blood+RL group. Those rats that had no resuscitation (HS group) or were resuscitated with blood/ blood+NS expired shortly after resuscitation.

Ratio of urinary NAG activity to urinary Cr level as a predictive biomarker in early kidney tubular injury in HS state

Mean level of urinary NAG, Cr level and NAG-Cr ratio are shown in Table 2. Urinary NAG level and NAG-Cr ratio were significantly higher in the HS+Blood+RL group compared to the Sham group (Table 2). Addition of EPO for resuscitation significantly decreased urinary NAG level and NAG-Cr ratio compared to the HS+Blood+RL group. Urinary Cr concentration showed attenuation in the HS+Blood+RL group compared to the Sham group. In the EPO group, urinary Cr concentration were significantly higher compared to the HS+Blood+RL group.

Assessment of renal NGAL $m R N A$ expression as a predictive biomarker of kidney damage in HS state

In this study, significant increase in renal NGAL mRNA expression in the HS+Blood+RL group was observed com-

Table 2. Effects of severe hemorrhagic shock and resuscitation with blood + Ringer's lactate with or without erythropoietin on changes in urinary NAG activity, urinary Cr concentration and NAG-Cr ratio in rats

\begin{tabular}{lccc}
\hline & \multicolumn{3}{c}{ Group } \\
\cline { 2 - 4 } & Sham & HS+Blood+RL & EPO \\
\hline Urinary NAG activity (U/l) & $50.24 \pm 10.48$ & $240.03 \pm 21.673^{*}$ & $103 \pm 11.74^{\star *}$ \\
Urinary Cr concentration $(\mathrm{mg} / 100 \mathrm{ml})$ & $47.5 \pm 3.24$ & $21.7 \pm 5.47^{*}$ & $42.33 \pm 5.14^{\#}$ \\
Urinary NAG activity/Urine Cr concentration $(\mathrm{U} / \mathrm{mg})$ & $0.1 \pm 0.02$ & $1.23 \pm 0.64^{*}$ & $0.27 \pm 0.04^{* \#}$ \\
\hline
\end{tabular}

Data are presented as the mean \pm SEM. HS, hemorrhagic shock; RL, Ringer's lactate; EPO, erythropoietin; NAG, N-acetyl- $\beta$-D-glucosaminidase; Cr, creatinine. ${ }^{*} p<0.05$ vs. Sham group, ${ }^{\#} p<0.05$ vs. HS+Blood+RL group. For more information, see Materials and Methods. 
pared to the Sham group (Fig. 2). EPO administration in resuscitation regimen significantly decreased renal NGAL gene expression compared to the HS+Blood+RL group.

Plasma enzymes activity for liver function evaluation in $H S$ state

In the Sham rats, surgical procedure did not result in any significant alterations in the plasma levels of AST and ALT (Table 1). In the HS+Blood+RL rats, plasma activities of AST and ALT significantly increased after the end of shock period compared to the Sham group. Plasma levels of AST and ALT were decreased by addition of EPO to the resuscitative fluids compared to the HS+Blood+RL group.

\section{Renal and liver histology}

In the Sham group, renal sections had no significant histological changes (Fig. 3A). After induction of HS, there were severe changes in the tissues compared to the Sham group such as frequent cast formation and obstruction in the tubules as well as tissue congestion and degeneration of the cells. Vascular congestion was also frequently present (Fig. 3B). EPO administration reduced the renal structural
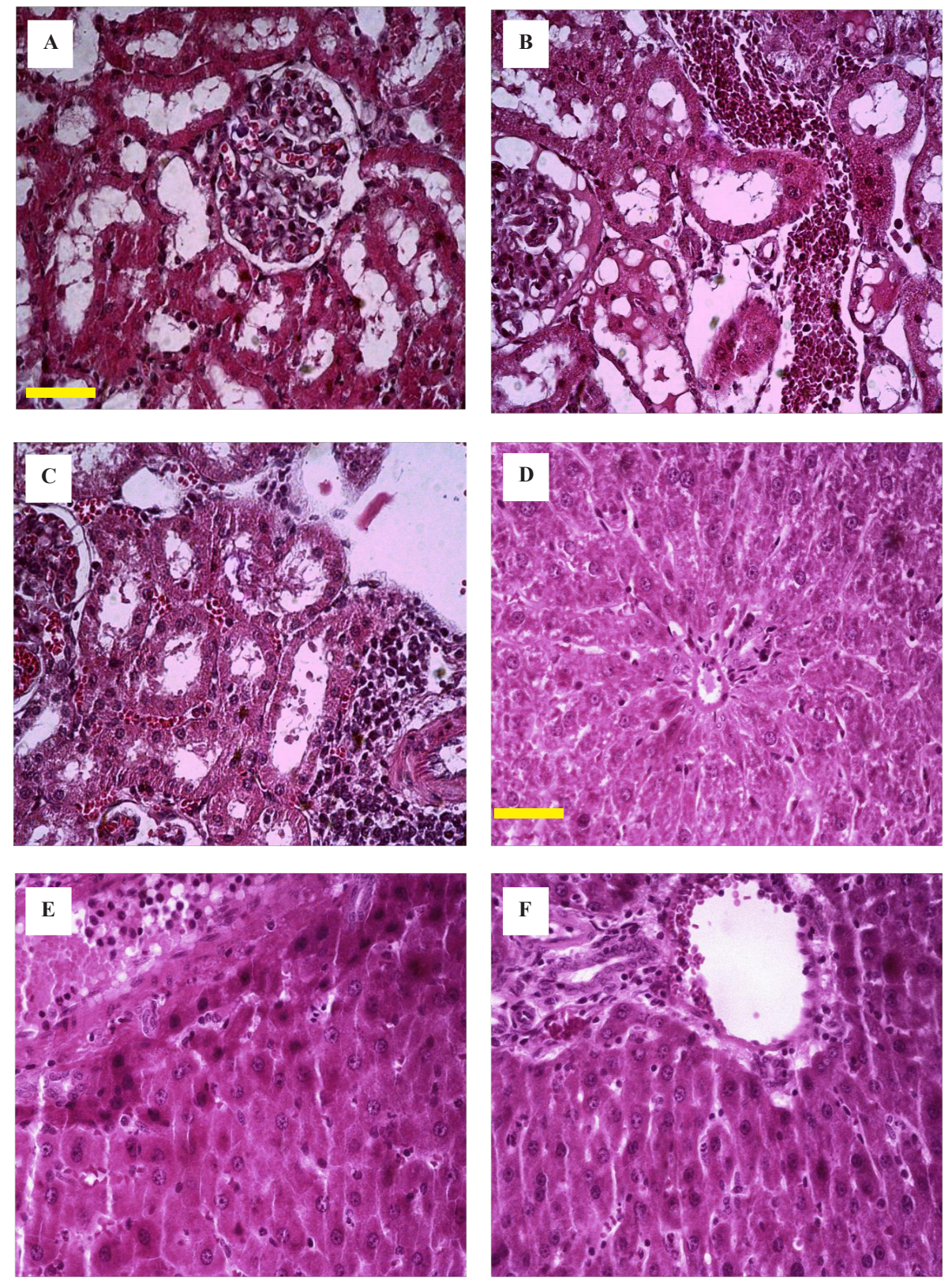

Figure 3. Effects of severe hemorrhagic shock and resuscitation with blood + Ringer's lactate with or without erythropoietin on the kidney $(\mathbf{A}, \mathbf{B}, \mathbf{C})$ and liver (D, E, F) histological changes. (A and D) Sham group, (B and E) HS+Blood+RL group, (C and F) EPO group. Magnification $\times 400$. Scale bar: $100 \mu \mathrm{m}$. For more information, see Materials and Methods. 
damages. Less cast formation in the tubules and less vascular congestion was observed (Fig. 3C).

Liver sections in the Sham group showed normal hepatic architecture (Fig. 3D). In the HS+Blood+RL group, there were changes in the tissues including interstitial edema, infiltration of leukocytes and severe sinusoidal and vascular congestion. In this group, pyknotic and atypical nuclei were observed (Fig. 3E). In the EPO group, cellular disintegration was less and sections were almost showing normal features including cellular and sinusoidal components (Fig. 3F).

\section{Oxidative stress markers in the kidney, liver and brain}

There was a significant increase in renal MDA level in the HS+Blood+RL group compared to the Sham group (Table 3 ). EPO significantly attenuated the rises in MDA content in comparison to the Sham group. Renal SOD activity was significantly attenuated in the HS+Blood+RL group compared to the Sham group (Table 3 ). SOD activity was significantly higher in the EPO group compared to the HS+Blood+RL group.

Liver MDA content was significantly increased in the HS+Blood+RL group compared to the Sham group. There was a significant decrease in liver MDA level in the EPO group compared to the HS+Blood+RL group. Liver SOD activity was significantly decreased in the HS+Blood+RL group compared to the Sham group. EPO administration increased SOD activity in the liver tissue samples compared to the HS+Blood+RL group.

Brain MDA level in the HS+Blood+RL group was significantly greater than the Sham group. EPO administration reduced brain MDA content compared to the $\mathrm{HS}+\mathrm{Blood}+\mathrm{RL}$ group. SOD activity was significantly lower in the brain tissue homogenates in the HS+Blood+RL group compared to the Sham group. There was a significantly higher SOD activity in the EPO group compared to the HS+Blood+RL group.

\section{Pro-inflammatory cytokines formation in the kidney, liver and brain}

In the HS+Blood+RL group, renal tissue tumor necrosis factor- $\alpha$ (TNF- $\alpha$ ) and interleukin- 6 (IL-6) were significantly increased compared to the Sham group (Table 3). EPO administration significantly prevented the rises in renal tissues TNF- $\alpha$ and IL- 6 compared to the HS+Blood+RL group.

These two cytokines also showed significant increases in the liver tissue homogenates compared to the Sham group. Addition of EPO to the resuscitative fluid led to a decrease in liver TNF- $\alpha$ and IL- 6 compared to the HS+Blood+RL group.

Hemorrhagic shock and resuscitation with blood plus RL resulted in a significant increase in brain TNF- $\alpha$ and IL- 6 levels. EPO administration decreased TNF- $\alpha$ and IL- 6 in the brain tissue samples compared to the HS+Blood+RL group.

\section{Discussion}

Although, many studies have recently been focused on animal models for HS induction, they are still lacking enough beneficial outcomes. Therefore, a fixed-volume hemorrhage approach was used in this study to create a severe, lifethreatening and reliable experimental HS model in rats.

Table 3. Effects of severe hemorrhagic shock and resuscitation with blood + Ringer's lactate with or without erythropoietin on oxidative stress indices and pro-inflammatory cytokines formation in the kidney, liver and brain tissue samples in rats

\begin{tabular}{|c|c|c|c|c|}
\hline & & \multicolumn{3}{|c|}{ Group } \\
\hline & & Sham & $\mathrm{HS}+$ Blood $+\mathrm{RL}$ & EPO \\
\hline \multirow{3}{*}{$\begin{array}{l}\text { MDA } \\
(\mu \mathrm{mol} / \mathrm{g} \text { tissue })\end{array}$} & Kidney & $27.48 \pm 2.06$ & $66.15 \pm 6.56^{*}$ & $35 \pm 9.24^{\#}$ \\
\hline & Liver & $35.42 \pm 4.84$ & $99.24 \pm 7.45^{\star}$ & $53.71 \pm 5.07^{\#}$ \\
\hline & Brain & $19.54 \pm 3.48$ & $45.18 \pm 4.42^{\star}$ & $29.66 \pm 5.16^{\#}$ \\
\hline \multirow{3}{*}{$\begin{array}{l}\text { SOD } \\
\text { (U/g tissue) }\end{array}$} & Kidney & $54.25 \pm 3.85$ & $5.036 \pm 2.16^{*}$ & $31.14 \pm 4.18^{\star \#}$ \\
\hline & Liver & $49.85 \pm 7.42$ & $9.91 \pm 1.15^{\star}$ & $23.19 \pm 3.79^{\star \#}$ \\
\hline & Brain & $67.15 \pm 6.45$ & $21.47 \pm 3.17^{\star}$ & $48 \pm 7.09^{\#}$ \\
\hline \multirow{3}{*}{$\begin{array}{l}\text { TNF- } \alpha \\
\text { (pg/g tissue) }\end{array}$} & Kidney & $13.29 \pm 2.99$ & $187.5 \pm 13.49^{*}$ & $79.01 \pm 8.41^{\star \#}$ \\
\hline & Liver & $19 \pm 5.18$ & $206.66 \pm 17.24^{*}$ & $85.47 \pm 12.15^{\star \#}$ \\
\hline & Brain & $11.5 \pm 2.03$ & $76 \pm 6.37^{\star}$ & $33.71 \pm 5.5^{\star \#}$ \\
\hline \multirow{3}{*}{$\begin{array}{l}\text { IL-6 } \\
\text { (pg/g tissue) }\end{array}$} & Kidney & $84.5 \pm 12.28$ & $885.77 \pm 52.17^{*}$ & $203.14 \pm 16.05^{\star \#}$ \\
\hline & Liver & $92.67 \pm 10.6$ & $1524 \pm 64.29^{*}$ & $167.18 \pm 17.11^{\star \#}$ \\
\hline & Brain & $54.5 \pm 0.95$ & $603 \pm 25.47^{\star}$ & $111.94 \pm 8.04^{\star \#}$ \\
\hline
\end{tabular}

Data are presented as the mean \pm standard error of mean. HS, hemorrhagic shock; RL, Ringer's lactate; EPO, erythropoietin; MDA, malondialdehyde; SOD, superoxide dismutase; TNF- $\alpha$, tumor necrosis factor- $\alpha$; IL-6, interleukin-6. ${ }^{\star} p<0.05 v$ s. Sham group, ${ }^{\#} p<0.05$ $v s$. HS+Blood+RL group. For more information, see Materials and Methods. 
In fixed-volume models, blood removal and hemorrhage is based on body weight. Since the heavier animals usually have more fat and therefore comparatively less blood, the circulating blood volume in mammalians does not correlate strictly with body weight (Ronn et al. 2011). To overcome this problem and increase the severity of HS, a narrower range of rat's body weight (285-300 g) was used.

To date, none of the several hemorrhagic shock-resuscitation models represented the severity and clinically relevant aspects. The model described here is closely orientated towards human clinical practice. The experimental plan of shock induction within 30 minutes, followed by a hemorrhagic shock period of $120 \mathrm{~min}$, and subsequent resuscitation for $30 \mathrm{~min}$ appears to mimic most urgent situations realistically.

During HS, urgent management of hemorrhage and proper fluid resuscitation is mandatory for maintaining microcirculation especially in vital organs. This is due to the fact that hypoxia in tissues may lead to systemic inflammatory response syndrome and multiple organ failure (Harlak et al. 2009). Hence, different resuscitative regimens were used and their effects on outcome and mortality in an animal model were compared.

More than half of the rats receiving blood and RL with or without EPO for resuscitation survived during entire experimental period, whereas those that had no resuscitation or were resuscitated with blood alone or blood plus NS expired shortly afterward. Thus, data analysis was performed between the Sham, HS+Blood+RL and EPO groups. HS+Blood + RL rats exhibited striking organ damage, as demonstrated histologically and indicated by considerable release of liver enzymes into plasma and increased plasma Cr concentration. They also showed rise in urinary NAG activity and renal NGAL mRNA expression. Moreover, HS also caused increases in oxidative stress parameters and cytokines formation in the kidney, liver and brain tissue samples compared to the Sham group. When EPO was added to the resuscitation regimen, it had beneficial effects on the survival rate, organ damage, renal and liver function as well as reducing oxidative stress markers and cytokines formation in the tissue samples.

In the current study of severe HS model, the mortality analysis indicated that resuscitation with the shed blood and addition of RL with or without EPO is more effective than other resuscitative regimens. The survival rates between the HS, HS+Blood and HS+Blood+NS groups were not significantly different. This indicates that blood resuscitation alone or in combination with NS has no benefit on survival rate.

There are several reports indicating that HS significantly increases plasma $\mathrm{Cr}$ concentration (Lee et al. 2012; Montegudo et al. 2013). In this study, rats that were resuscitated with blood plus RL had higher plasma Cr level and showed severe changes in the renal tissues compared to the Sham group. EPO is suggested to attenuate the rises in plasma $\mathrm{Cr}$ in HS state (Wu et al. 2010; Nandra et al. 2013). Here, resuscitation with blood plus RL in combination with EPO reduced the HS-induced increase in plasma Cr level.

Although acute kidney injury (AKI) is clinically diagnosed by serum $\mathrm{Cr}$ level, but, it is a non-reliable marker during acute changes in kidney function (Devarajan 2008). However, in recent years, two early predictive and non-invasive AKI biomarkers, namely NAG and NGAL, have been suggested (Mishra et al. 2004; Skálová 2005; Devarajan 2008).

In normal conditions, low concentrations of NAG enzyme are detectable in healthy human urine, but these levels increase significantly after renal injury. Urinary NAG activity is known to be a sensitive marker of early kidney tubular injury (Seifi et al. 2014) and it precedes changes in the plasma Cr level (Skálová 2005). As mentioned above, the urinary NAG values should be expressed as a ratio to urinary $\mathrm{Cr}$ concentration (Supavekin et al. 2003). Our findings showed that urinary NAG level and NAG-Cr ratio were significantly higher in the HS+Blood+RL group compared to the Sham group. EPO administration attenuated the rises in NAG level and NAG-Cr ratio. Since the urinary excretion of NAG is proportional to the degree of renal injury, it is concluded that EPO may be capable of reducing kidney damage.

NGAL is one of the most upregulated genes in the early renal ischemia-reperfusion injury (Devarajan 2008). Notably, NGAL protein is easily identified in the blood and urine immediately after AKI (Mishra et al. 2003). In this study, $\mathrm{HS}+\mathrm{Blood}+\mathrm{RL}$ rats showed a significant increase in renal NGAL gene expression, indicating AKI induction following HS. EPO decreased NGAL mRNA expression induced by HS in renal tissue samples.

The effects of resuscitation with Blood+RL with or without EPO on liver function after HS induction were also examined. Animals in the HS+Blood+RL group showed significant rises in the plasma levels of AST and ALT compared to the Sham group. EPO attenuated the rises in AST and ALT levels compared to the HS+Blood+RL group. This data provided evidence for the development of liver injury in this model of HS and resuscitation and indicated that EPO is able to reduce hepatocellular damage.

The other complication of HS is thought to be related to the activation of the immune system. Under hypoxic conditions, changes in the activity of pro-inflammatory pathways initiate systemic inflammatory response which may lead to multiple organ failure (Harlak et al. 2009). During hemorrhage, the inflammatory cascade is stimulated by many factors including formation of free radicals and substantial amounts of TNF- $\alpha$ and IL-6 (Wu et al. 2010). These cytokines have been verified to reach to their maximum levels early after HS (Wu et al. 2010). There are reports that ischemiareperfusion injury induces TNF- $\alpha$ production in different organs (Donnahoo et al. 1999). In this study, HS+Blood+RL 
rats showed a significant increase in TNF- $\alpha$ and IL- 6 levels in the kidney, liver and brain tissue samples compared to the Sham rats. It is suggested that these cytokines coordinate an inflammatory response between blood cells, vascular endothelium, and resident cells in different tissues which ultimately lead to synthesis of chemokines, cell adhesion molecules and aggravation of organ damage (Donnahoo et al. 1999). EPO is suggested to act as an anti-inflammatory agent which reduces oxidative stress by stimulating cell survival pathways (Kumar and Bhoi 2015). When EPO was administered into the resuscitation fluid, it significantly lowered cytokines levels. These results confirm that EPO in the resuscitation fluid exerts protective effects on tissue damage by reducing pro-inflammatory elements.

In the present study, blood was administered alone or in combination with NS, RL or RL+EPO for resuscitation. The results of mortality rate as presented above demonstrated a distinct benefit of resuscitation with RL. When EPO was added to RL, the beneficial effects were more evident. Although some studies have reported beneficial effects with blood alone resuscitation (Onen et al. 2003), it had no useful effects. Findings are consistent with Wang et al. (2015) demonstrating that blood transfusion alone induced multiple organ failure, evidenced by a high scores of lung, kidney and liver damages. Kiraly and co-workers reported that resuscitation with RL improved hemodynamic parameters, blood coagulation, and survival rate compared to the resuscitation with NS in an uncontrolled hemorrhagic shock swine model (Kiraly et al. 2006). Early studies on hemorrhagic shock have also shown advantageous outcomes in the resuscitation with RL (Cervera and Moss 1975; Healey et al. 1998). It seems that different outcomes in the mortality using NS or RL are related to the involvement of two factors. First, physiological concentrations of sodium and calcium may contribute to the higher beneficial effects of resuscitation with RL (Rohrig et al. 2012). In most of the other studies, moderate types of hemorrhagic shock were induced. However, in this study, resuscitation with blood plus RL didn't have enough beneficial effect on early organ injury. Neither functional markers nor oxidative stress and cytokines formation parameters recovered after resuscitation with shed blood plus RL solution and multiple organ failure in resuscitation with blood and RL was observed. This apparent discrepancy is most likely a result of the severity of the HS model. Thus, the degree of the severity of the model is the second important factor and it seems that the lack of a moderate HS model was one of the limitations of the current study.

Here, the tissue protective actions of EPO were demonstrated when added to the resuscitation fluids. In the other studies, EPO was injected before HS induction (Ranjbaran et al. 2017a) or resuscitation but this is the first time that EPO was administered in combination to RL for resuscitation. As mentioned above, EPO reduced the renal dysfunction, liver and brain injury caused by HS. This suggests EPO is an effective agent that increases the resistance of tissues and organs to withstand during HS state. It is suggested that EPO is able to increase basal blood pressure and improve tissue perfusion (Nandra et al. 2013). Abdelrahman et al. showed that the beneficial effects of EPO was related to the prevention of apoptosis process by reducing caspase 3, 8 and 9 activities (Abdelrahman et al. 2004). It is hypothesized that EPO activates several intracellular signaling pathways, in particular JAK/STAT, PI3K/Akt and MAPK pathways and stimulates survival signals (Nandra et al. 2013; Kumar and Bhoi 2015). We have previously reported that administration of EPO (300 IU/kg, i.v.) $10 \mathrm{~min}$ prior to HS induction, reduced renal dysfunction by activation of survival pathway (activated Akt pathway) and reduction in the apoptotic process (reduced caspase 3 activity) (Ranjbaran et al. 2017b). However, the exact mechanisms mediating these beneficial effects of EPO on tissues are not fully clear and warrant further research.

It should be noticed that resuscitation with RL solution was relatively more effective than blood or NS in rats, RL lowered mortality rate but was not effective on ischemic damage and the subsequent kidney, liver and brain tissue injuries. The absence of prevention in organ damage may be related to the severity of HS. RL may exert positive effects on organs in mild or moderate models of HS. We suggest that EPO administration in combination to blood and RL is capable of attenuating organ injury and dysfunction through reducing oxidative stress and pro-inflammatory indices associated with HS. Thus, addition of EPO to RL as a resuscitative therapy may be a promising way to prevent organ damage in severe HS.

Acknowledgements. This work was supported by a grant from Tehran University of Medical Sciences.

Conflict of interest. The authors declare that they have no conflict of interest.

\section{References}

Abdelrahman M, Sharples EJ, Mcdonald MC, Collin M, Patel NSA, Yaqoob MM, Thiemermann C (2004): Erythropoietin attenuates the tissue injury associated with hemorrhagic shock and myocardial ischemia. Shock 22, 63-69 https://doi.org/10.1097/01.shk.00001276869.21260.9d

Ahmadi-Yazdi C, Williams B, Oakes S, Moore FD Jr. (2009): Attenuation of the effects of rat hemorrhagic shock with a reperfusion injury-inhibiting agent specific to mice. Shock 32, 295-301 https://doi.org/10.1097/SHK.0b013e3181995e0c

Ali RJ, Al-Obaidi FH, Arif HS (2014): The role of urinary N-acetyl Beta-D-glucosaminidase in children with urological problems. Oman Med. J. 29, 285-288 https://doi.org/10.5001/omj.2014.74 
Boldt J (2004): Fluid choice for resuscitation of the trauma patient: a review of the physiological, pharmacological, and clinical evidence. Can. J. Anaesth. 51, 500-513 https://doi.org/10.1007/BF03018316

Borovnik-Lesjak V, Whitehouse K, Baetiong A, Miao Y, Currie BM, Velmurugan S, Radhakrishnan J, Gazmuri RJ (2014): Effects of intraosseous erythropoietin during hemorrhagic shock in swine. PLoS One 9, 1-15 https://doi.org/10.1371/journal.pone.0110908

Cervera AL, Moss G. (1975): Dilutional re-expansion with crystalloid after massive hemorrhage: saline versus balanced electrolyte solution for maintenance of normal blood volume and arterial pH. J. Trauma. 15, 498-503 https://doi.org/10.1097/00005373-197506000-00008

Devarajan P (2008): Neutrophil gelatinase-associated lipocalin (NGAL): A new marker of kidney disease. Scand. J. Clin. Lab. Invest. Suppl. 241, 89-94 https://doi.org/10.1080/00365510802150158

Donnahoo KK, Shames BD, Harken AH, Meldrum DR (1999): Review article: the role of tumor necrosis factor in renal ischemia-reperfusion injury. J. Urol. 162, 196-203 https://doi.org/10.1097/00005392-199907000-00068

Esterbauer H, Cheeseman K (1990): Determination of aldehydic lipid peroxidation products: Malonaldehyde and 4-hydroxynonenal. Methods Enzymol. 186, 407-421 https://doi.org/10.1016/0076-6879(90)86134-H

Fülöp A, Turóczi Z, Garbaisz D, Harsányi L, Szijártó A (2013): Experimental models of hemorrhagic shock: a review. Eur. Surg. Res. 50, 57-70 https://doi.org/10.1159/000348808

Harlak A, Fatih Can M, Mentes O, Ersoz N, Kurt B, Turker T, Yagci G, Tufan T (2009): Does the type of resuscitative fluid affect healing of colonic anastomosis in experimentally induced hemorrhagic shock in rats? Med. Princ. Pract. 18, 255-260 https://doi.org/10.1159/000215720

Healey MA, Davis RE, Liu FC, Loomis WH, Hoyt DB (1998): Lactated ringer's is superior to normal saline in a model of massive hemorrhage and resuscitation. J. Trauma. 45, 894-899 https://doi.org/10.1097/00005373-199811000-00010

Kiraly LN, Differding JA, Enomoto TM, Sawai RS, Muller PJ, Diggs B, Tieu BH, Englehart MS, Underwood S, Wiesberg TT, Schreiber MA (2006): Resuscitation with normal saline (NS) vs. lactated Ringer's (LR) modulates hypercoagulability and leads to increased blood loss in an uncontrolled hemorrhagic shock swine model. J. Trauma. 61, 57-64 https://doi.org/10.1097/01.ta.0000220373.29743.69

Knotzer H, Pajk W, Maier S, Du“Nser MW, Ulmer H, Schwarz B, Salak N, Hasibeder WR (2006): Comparison of lactated Ringer's, gelatine and blood resuscitation on intestinal oxygen supply and mucosal tissue oxygen tension in haemorrhagic shock. Br. J. Anaesth. 94, 509-516 https://doi.org/10.1093/bja/ael208

Kumar M, Bhoi S (2015): Does erythropoietin reactivate bone marrow dysfunction in trauma hemorrhagic shock? Int. J. Crit. Illn. Inj. Sci. 5, 230-231 https://doi.org/10.4103/2229-5151.170848

Lee RP, Lee CJ, Subeq YM, Peng TC, Yang FL, Hsu BG (2012): Erythropoietin ameliorates severe hemorrhagic shock-induced serum proinflammatory cytokines and biochemical changes in spontaneously hypertensive rats. Tzu Chi Medical Journal 24, $46-50$ https://doi.org/10.1016/j.tcmj.2012.04.004

Mishra J, Ma Q, Prada A, Mitsnefes M, Zahedi K, Yang J (2003): Identification of neutrophil gelatinaseassociated lipocalin as a novel early urinary biomarker for ischemic renal injury. J. Am. Soc. Nephrol. 4, 2534-2543 https://doi.org/10.1097/01.ASN.0000088027.54400.C6

Mishra J, Mori K, Ma Q, Kelly C, Barasch J, Devarajan P (2004): Neutrophil gelatinase-associated lipocalin: a novel early urinary biomarker for cisplatin nephrotoxicity. Am. J. Nephrol. 24, 307-315 https://doi.org/10.1159/000078452

Montegudo AE, Palilonis MA, Moore CC, Huynh T, Mckillop IH, Evans SL (2013): Pyrrolidine dithiocarbamate improves mortality in a rat model of severe hemorrhage. J. Surg. Res. 179, E149-155 https://doi.org/10.1016/j.jss.2012.01.006

Nandra KK, Collino M, Rogazzo M, Fantozzi R, Patel NSA, Thiemermann C (2013): Pharmacological preconditioning with erythropoietin attenuates the organ injury and dysfunction induced in a rat model of hemorrhagic shock. Dis. Model Mech. 6, 701-709 https://doi.org/10.1242/dmm.011353

Onen A, Cigdem MK, Deveci E, Kaya S, Turhanoglu S, Yaldiz M (2003): Effects of whole blood, crystalloid, and colloid resuscitation of hemorrhagic shock on renal damage in rats: an ultrastructural study. J. Pediatr. Surg. 38, 1642-1649 https://doi.org/10.1016/S0022-3468(03)00572-4

Paoletti F, Mocali A (1988): Changes in cuzn-superoxide dismutase during induced differentiation of murine erythroleukemia cells. Cancer Res. 48, 6674-6677

Ranjbaran M, Kadkhodaee M, Seifi B (2017a): Renal tissue proinflammatory gene expression is reduced by erythropoietin in rats subjected to hemorrhagic shock. J. Nephropathol. 6, 69-73 https://doi.org/10.15171/jnp.2017.12

Ranjbaran M, Kadkhodaee M, Seifi B, Adelipour M, Azarian B (2017b): Erythropoietin attenuates experimental haemorrhagic shock-induced renal damage through an iNOS- dependent mechanism in male Wistar rats. Injury 48, 262-269 https://doi.org/10.1016/j.injury.2017.01.010

Rohrig R, Ronn T, Lendemans S, Feldkamp T, Groot HD, Petrat F (2012): Adverse effects of resuscitation with lactated Ringer compared with Ringer solution after severe hemorrhagic shock in rats. Shock 38, 137-145 https://doi.org/10.1097/SHK.0b013e31825b4ed9

Ronn T, Lendemans S, De Groot H, Petrat F (2011): A new model of severe hemorrhagic shock in rats. Comp. Med. 61, 419-426

Seifi B, Kadkhodaee M, Bakhshi E, Ranjbaran M, Ahghari P, Rastegar T (2014): Enhancement of renal oxidative stress by injection of angiotensin II into the paraventricular nucleus in renal ischemia-reperfusion injury. Can. J. Physiol. Pharmacol. 2, 752-757 https://doi.org/10.1139/cjpp-2014-0108

Skálová S (2005): The diagnostic role of urinary N-acetyl BetaD-glucosaminidase (NAG) activity in the detection of renal tubular impairment. Acta Medica 48, 75-80 
Supavekin S, Zhang W, Kucherlapati R, Kaskel FJ, Moore LC, Devarajan P (2003): Differential gene expression following early renal ischemia/reperfusion. Kidney Int. 63, 1714-1724 https://doi.org/10.1046/j.1523-1755.2003.00928.x

Wang CH, Lin HJ, Hsu CC, Huang HS (2015): Resuscitation with whole body cooling prevents multiple organ damage after hemorrhagic shock in rats. FASEB J. 29 Supplement, 641.5
Wu WT, Lin NT, Subeq YM, Lee RP, Chen IH, Hsu BG (2010): Erythropoietin protects severe haemorrhagic shock-induced organ damage in conscious rats. Injury 41, 724-730 https://doi.org/10.1016/j.injury.2009.12.006

Received: January 2, 2017

Final version accepted: May 3, 2017 